\title{
Submucosal tunneling endoscopic resection for leiomyomas located on opposite esophageal walls: the role of a tortuous submucosal tunnel
}

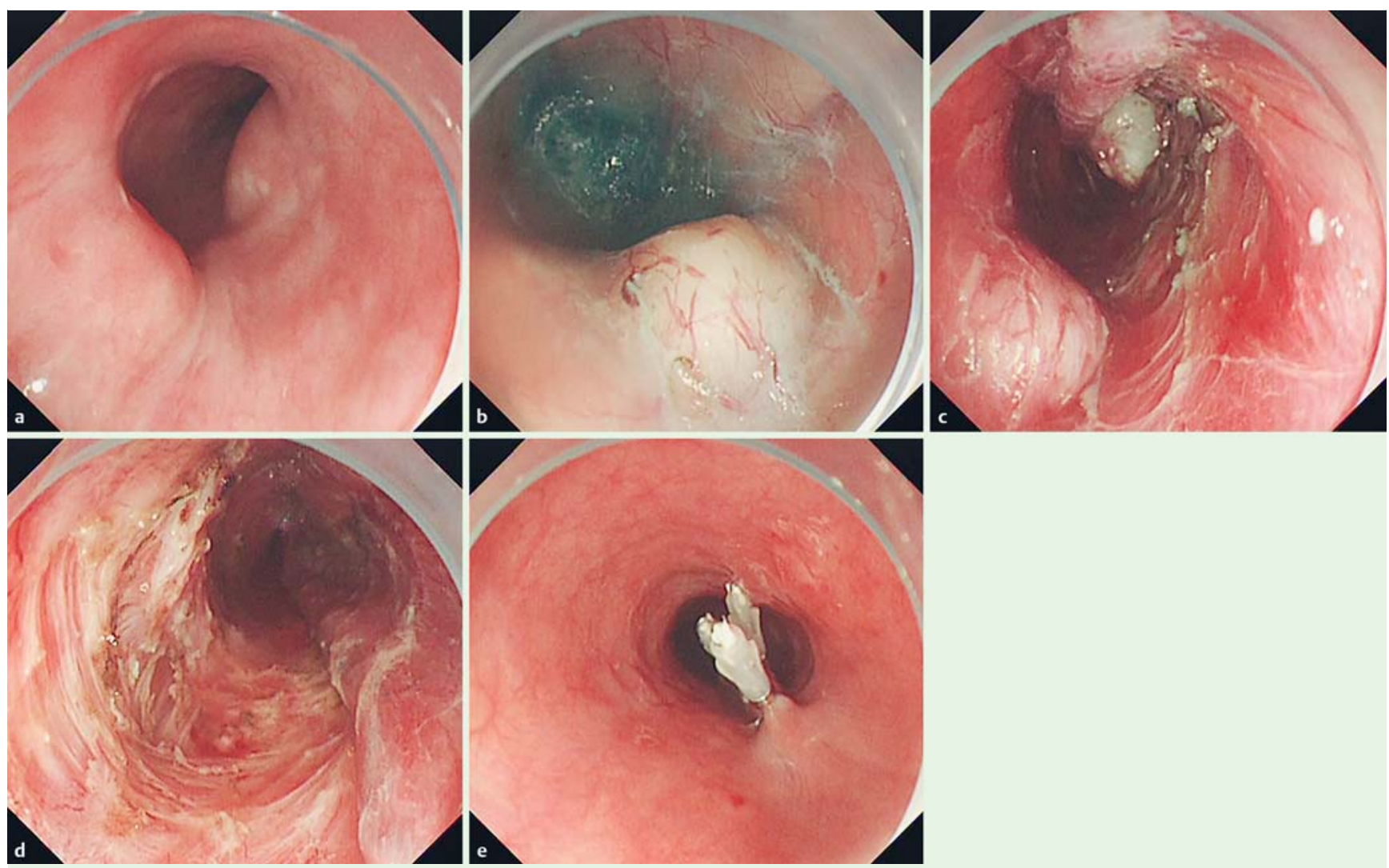

Fig. 1 Endoscopic views showing: a two protruding lesions in the esophagus; $\mathbf{b}$ the first tumor in the submucosal tunnel; $\mathbf{c}$ the second tumor within the same submucosal tunnel; $\mathbf{d}$ the wound surface after removal of the two tumors; $\mathbf{e}$ the mucosal entry point closed with several clips.

A 50-year-old man presented to our hospital with a 1-month history of epigastric discomfort. Esophagogastroduodenoscopy (EGD) revealed two protruding lesions on the anterior and posterior esophageal walls, about $27 \mathrm{~cm}$ and $30 \mathrm{~cm}$ from the incisors, respectively ( $\bullet$ Fig. 1 a; $\bullet$ Video 1 ). Endoscopic ultrasonography (EUS) revealed that the tumors were originating from the muscularis propria layer and had no risk features.

Submucosal tunneling endoscopic resection (STER) was performed. After a longitudinal mucosal incision had been made, a submucosal tunnel was created and the first submucosal tumor (SMT) could be seen ( $\checkmark$ Fig. 1 b). As the two tumors were located on opposite walls of the esophagus, it was impossible to locate the second tumor using purely a straight submucosal tunnel, so a tortuous tunnel was created. Submucosal injection of methylene blue was performed to preset the tunnel route, and the two separate tumors were identified, with a distance of about $3 \mathrm{~cm}$ between them ( Fig. 1 c). The tumors were carefully dissected off the muscularis propria layer ( $\bullet$ Fig.1d), and the mucosal entry was closed ( $\bullet$ Fig. 1 e; Video 2 ). The STER procedure was completed uneventfully within 80 minutes. The diameters of the resected SMTs were $2 \mathrm{~cm}$ and $1.5 \mathrm{~cm}(\bullet \mathrm{Fig} .2)$ and they were both leiomyomas histopathologically.

STER has been demonstrated to be safe and effective for treating upper gastrointestinal SMTs [1,2]. There have been only a few reports regarding STER for multiple SMTs, and the tumors in each of the reported cases were in the same or adjacent parts of the esophageal/gastric wall, so a single submucosal tunnel was sufficient to remove the tumors [3-5]. In the present case, the two SMTs were found on opposite

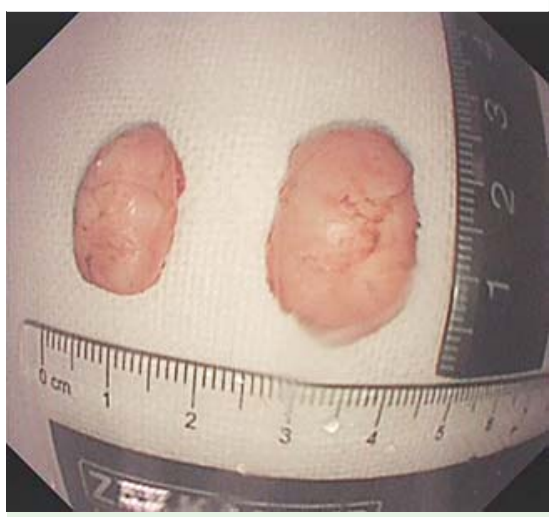

Fig. 2 Macroscopic appearance of the two resected tumors.

esophageal walls, and at first two submucosal tunnels were recommended. This however would mean excessive submucosal dissection and two tunnel entries, which, as well as being time-consuming, 


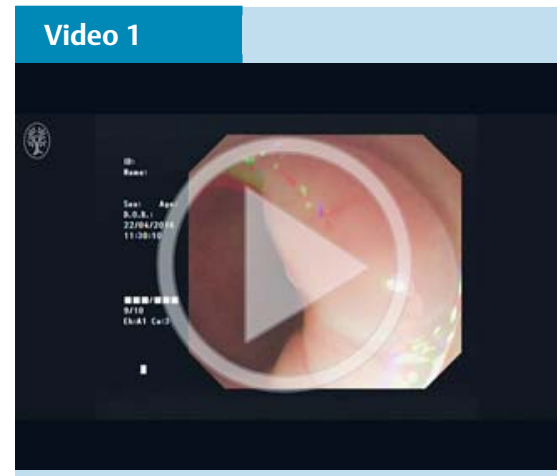

Endoscopy showing two protruding lesions on opposite walls of the esophagus.

would increase the complication rate. We successfully removed both of the tumors by STER within a single tunnel by creating a tortuous submucosal tunnel.

Endoscopy_UCTN_Code_TTT_1AO_2AG

\section{Competing interests: None}

\section{Fuxi Zhou, Yuyong Tan, Yi Chu,} Changmei Hu, Jirong Huo, Deliang Liu

Department of Gastroenterology, The Second Xiangya Hospital of Central South University, Changsha, Hunan, China

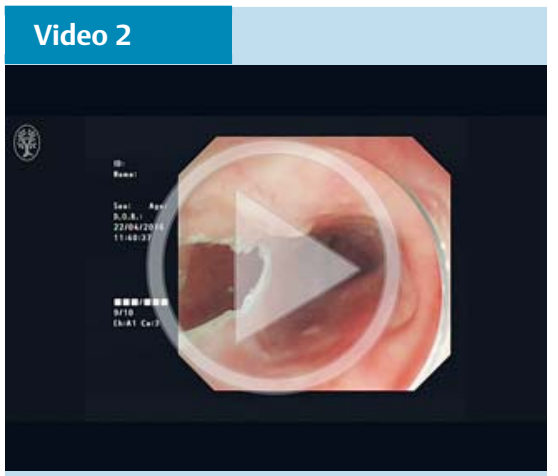

The submucosal tunneling endoscopic resection (STER) procedure being performed for two tumors on opposite esophageal walls.

\section{References}

1 Chen T, Zhou PH, Chu Y et al. Long-term outcomes of submucosal tunneling endoscopic resection for upper gastrointestinal submucosal tumors. Ann Surg 2016. Epub ahead of print 2016 Jan 19

2 Wang H, Tan Y, Zhou Yet al. Submucosal tunneling endoscopic resection for upper gastrointestinal submucosal tumors originating from the muscularis propria layer. Eur J Gastroenterol Hepatol 2015; 27: 776-780

3 Chen $\mathrm{H}, \mathrm{Xu} \mathrm{Z}$, Huo J et al. Submucosal tunneling endoscopic resection for simultaneous esophageal and cardia submucosal tumors originating from the muscularis propria layer (with video). Dig Endosc 2015; 27: $155-158$
4 Zhang $C, H u$ JW, Chen $T$ et al. Submucosal tunneling endoscopic resection for upper gastrointestinal multiple submucosal tumors originating from the muscular propria layer: a feasibility study. Indian J Cancer 2015; 51 (Suppl. 02): e52 - e55

5 Tan Y, Hu C, Zhou Yet al. Submucosal tunneling endoscopic resection for multiple esophageal leiomyomas. Endoscopy 2016; 48 (Suppl. 01): E234 -E235

\section{Bibliography}

DOI http://dx.doi.org/

10.1055/s-0042-111323

Endoscopy 2016; 48: E255-E256

(c) Georg Thieme Verlag KG

Stuttgart · New York

ISSN 0013-726X

\section{Corresponding author \\ Deliang Liu, MD}

Department of Gastroenterology

The Second Xiangya Hospital of Central South

University

NO. 139 Middle Renmin Road

Changsha

Hunan, 410011

China

Fax: 86-0731-85533525

liudeliang@medmail.com.cn 